\title{
Analysis of Serum Ferritin Level and Its Clinical Value in Advanced Gastric Cancer
}

\author{
Xiaoyu Huang, Qi Wang, Qiwei Chen, Jingya Ye, Gong Chen, Qibing Wu* \\ Department of Radiotherapy, The First Affiliated Hospital of Anhui Medical University, Hefei, China \\ Email: *wqb71vip@163.com
}

How to cite this paper: Huang, X.Y., Wang, Q., Chen, Q.W., Ye, J.Y., Chen, G. and Wu, Q.B. (2022) Analysis of Serum Ferritin Level and Its Clinical Value in Advanced Gastric Cancer. Journal of Biosciences and Medicines, 10, 1-6.

https://doi.org/10.4236/jbm.2022.101001

Received: November 9, 2021

Accepted: January 4, 2022

Published: January 7, 2022

Copyright $\odot 2022$ by author(s) and Scientific Research Publishing Inc. This work is licensed under the Creative Commons Attribution International License (CC BY 4.0).

http://creativecommons.org/licenses/by/4.0/

\begin{abstract}
Objective: To investigate the concentration and clinical significance of serum ferritin (SF) in patients diagnosed with advanced gastric cancer before and after treatment. Methods: Forty patients with advanced gastric cancer diagnosed by cytology or pathology in our hospital were selected, including 25 males and 15 females, aged from 48 to 85 years, and the median age was 61.0 years. 40 healthy volunteers matched with age and education were selected as the control group. In order to study the changes of SF level in the treatment of advanced gastric cancer, we divided the patients into effective group (efficacy evaluation as partial remission or complete remission), ineffective group (efficacy evaluation as no remission) and recurrence group according to the efficacy after treatment. Then the difference of SF level between different groups and the relationship between SF level and curative effect were analyzed. There was no significant difference in gender and age among all groups. Results: The SF levels in the newly diagnosed group, stage III patient group and stage IV patient group were significantly higher than those in the control group. The level of SF in stage IV patients was significantly higher than that in stage III patients. There were significant differences in SF level between the effective treatment group, the newly diagnosed group and the ineffective treatment group, but there was no significant difference in SF level between the newly diagnosed group and the ineffective treatment group. Conclusion: SF level has a certain value in the auxiliary diagnosis of gastric cancer, and it also has a certain guiding significance for the evaluation of curative effect and prognosis after treatment.
\end{abstract}

\section{Keywords}

Gastric Cancer, Serum Ferritin, Diagnosis, Curative Effect 


\section{Introduction}

Gastric cancer is one of the most common malignant tumors in the world. Although its morbidity and mortality have declined in recent years, the incidence rate of gastric cancer remains the fifth in all malignant tumors, with the incidence rate of third [1]. With the continuous development of biotechnology, tumor markers such as antigen and protease produced by tumor cells have been gradually used in the clinical diagnosis and efficacy evaluation of tumor diseases. Serum ferritin (SF) is an iron related protein, which is normally used to measure the body's iron reserve. However, SF will also increase in some specific cases (such as acute and chronic inflammation, some infectious diseases and rheumatic diseases), despite the body does not have excessive iron reserves.

Using tumor markers to diagnose and screen patients with gastric cancer and monitor their condition has become one of the indispensable means of gastric cancer examination. At present, the markers used to detect gastric cancer are non-specific tumor related antigens, which mostly have the shortcomings of low specificity and sensitivity. At the same time, the joint detection of several specific or sensitive indicators can improve the sensitivity and specificity of gastric cancer diagnosis. Studies have found that the increase of SF often indicates the disease progression and shortened survival time of tumor patients, and also has a certain relationship with the prognosis [2]. In addition, many studies have also found that SF level is often related to tumor load and disease severity [3] [4]. However, only few studies on the diagnosis and efficacy evaluation of SF in patients with advanced gastric cancer. This study retrospectively analyzed the changes of SF level in 40 patients with newly diagnosed advanced gastric cancer before and after treatment. Then compared with the healthy control group, so as to provide some guidance for judging the prognosis of gastric cancer.

\section{Materials and Methods}

\subsection{Patient Data}

40 subjects diagnosed with advanced gastric cancer were selected as gastric cancer group who were treated in Hefei second people's Hospital from January 2015 to September 2021. All patients had cytological or pathological evidence. There were 25 males and 15 females. The age ranged from 48 to 85 years, and the median age was 61.0 years. Tumor location includes gastric fundus in 5 cases, gastric body in 21 cases, gastric antrum in 14 cases. Tissue differentiation includes 2 cases with highly differentiated, 14 cases with moderately differentiated and 24 cases with poorly differentiated. TNM stage includes stage IIIa in 23 cases, stage IIIb in 12 cases and stage IV in 5 cases.

In order to study the changes of SF level in the treatment of advanced gastric cancer, we divided the patients into treatment effective group (efficacy evaluation as partial remission or complete remission), treatment ineffective group (efficacy evaluation as no remission) and recurrence group according to the effica- 
cy after treatment. There was no significant difference in gender and age among all groups. The clinical data of patients are shown in Table 1.

We also selected 40 healthy people as healthy control group. All subjects in the healthy control group had no evidence of acute or chronic inflammation, infectious diseases, autoimmune diseases and tumor diseases. There were 25 males and 15 females. The age ranged from 51 to 82 years, and the median age was 60.0 years. There was no significant difference in the above general data between gastric cancer group and healthy control group $(\mathrm{P}>0.05)$.

\subsection{Determination of Serum Ferritin Level}

The chemiluminescence method is used for determination. The reagent is provided by Abbott Pharmaceutical Co. Ltd. and the operation is carried out according to the instructions. The ferritin normal reference value is $23.9-336.2$ $\mathrm{ng} / \mathrm{ml}$.

\subsection{Statistical Methods}

SPSS 18.0 statistical software was used for statistical analysis. The measurement data in line with normal distribution were expressed by $\mathrm{x} \pm \mathrm{s}$, and $\mathrm{t}$-test was used for comparison between groups. Chi square test was used for counting data. P < 0.05 was regarded as statistically significant.

\section{Results}

\subsection{SF Level in Newly Diagnosed Advanced Gastric Cancer Group and Healthy Control Group}

We measured the SF level in 40 newly diagnosed patients with gastric cancer. Patients with gastric cancer were divided into stage III patient group and stage IV patient group according to different TNM stages. The results showed that the SF levels in the newly diagnosed group, stage III patient group and stage IV patient group were significantly higher than those in the healthy control group. The level of SF in stage IV patients was significantly higher than that in stage III patients. The differences were statistically significant (see Table 2).

Table 1. General clinical data of patient group and healthy control group.

\begin{tabular}{cccc}
\hline Group & $\begin{array}{c}\text { Number } \\
\text { of cases }\end{array}$ & $\begin{array}{c}\text { Median age } \\
\text { (years) }\end{array}$ & $\begin{array}{c}\text { Gender } \\
\text { (male/female) }\end{array}$ \\
\hline $\begin{array}{c}\text { New } \\
\text { diagnosed }\end{array}$ & 40 & 61.0 & $25 / 15$ \\
Effective & 15 & 58.2 & $9 / 6$ \\
Ineffective & 25 & 62.5 & $14 / 11$ \\
Recurrence & 10 & 63.2 & $5 / 5$ \\
$\begin{array}{c}\text { Healthy } \\
\text { control }\end{array}$ & 40 & 60.0 & $25 / 15$ \\
\hline
\end{tabular}


Table 2. SF levels in patients with advanced gastric cancer and healthy controls.

\begin{tabular}{ccc}
\hline Group & Number of cases & SF $(\mathrm{ng} / \mathrm{ml})$ \\
\hline New diagnosed & $40^{*}$ & $473.3 \pm 119.4$ \\
stage III & $22^{\star *}$ & $391.3 \pm 64.5$ \\
stage IV & $18^{\star}$ & $540.4 \pm 112.5$ \\
Healthy control & 40 & $123.2 \pm 52.2$ \\
\hline
\end{tabular}

${ }^{\star} \mathrm{P}<0.05$, compared with the healthy control group; ${ }^{*} \mathrm{P}<0.05$, compared with stage III and stage IV initial diagnosis group.

\subsection{Relationship between SF Level and Efficacy Evaluation in Patients with Advanced Gastric Cancer}

We also analyzed the relationship between SF level and efficacy evaluation of advanced gastric cancer patients after treatment. The results showed that there were significant differences in SF level between the treatment effective group, the first diagnosis group and the treatment ineffective group. However, there was no significant difference in SF level between the new diagnosis group and the treatment ineffective group (see Table 3).

\section{Discussion}

Gastric cancer is one of the common malignant tumors in China. Early diagnosis is difficult because of its lack of specific symptoms and signs. However, the condition is often in the middle and late stage and the best treatment opportunity is always lost when patients have obvious clinical symptoms [5]. Therefore, early detection, early diagnosis and early treatment of gastric cancer are important measures to improve the survival rate of gastric cancer. Iron plays a key role in the growth and development of human normal cells. At the same time, it is also one of the key elements necessary for tumor cells to synthesize DNA. SF is an iron rich protein and the main form of iron storage in the body. Ferritin can promote the production of reactive oxygen species, produce too many oxygen free radicals, activate a variety of enzymes, denature nucleic acids and proteins, and lead to the mutation of tumor suppressor gene and DNA repair gene. Therefore, its main physiological function is to participate in cell metabolism, cell proliferation and immune regulation. Although ferritin levels are closely related to the level of iron storage, ferritin levels can also be elevated without iron overload in many diseases, such as inflammation, infectious diseases, organ dysfunction or malignancy [6]. It has been found that ferritin is generally highly expressed in tumor associated macrophages. Its elevated concentration is often closely related to tumor cell proliferation and drug resistance [7]. Moreover, iron overload can not only promote the growth of tumor cells, but also inhibit their apoptosis [8]. At present, many clinical studies have found that the level of SF is increased in patients with newly diagnosed malignant tumors. This study also found that the SF level was significantly increased in newly diagnosed patients with advanced gastric cancer. 
Table 3. Relationship between SF level and curative effect in patients with gastric cancer after treatment.

\begin{tabular}{ccc}
\hline Group & Number of cases & SF $(\mathrm{ng} / \mathrm{ml})$ \\
\hline New diagnosed & $40^{\#}$ & $473.3 \pm 119.4$ \\
effective & $15^{\star \#}$ & $258.2 \pm 105.9$ \\
ineffective & $25^{\#}$ & $635.7 \pm 152.0$ \\
Healthy control & 40 & $123.2 \pm 52.2$ \\
\hline
\end{tabular}

${ }^{\star} \mathrm{P}<0.05$, compared with the newly diagnosed group; ${ }^{*} \mathrm{P}<0.05$, compared with the healthy control group.

In order to explore the relationship between the elevated level of SF and the severity of newly diagnosed gastric cancer patients, we divided advanced gastric cancer patients into two groups according to the different disease stages at the time of diagnosis. The results showed that the level of TNM in different clinical stages of gastric cancer increased with the increase of tumor stage. In addition, we also analyzed the relationship between SF level and curative effect after treatment. We found that the SF level in the effective treatment group was significantly lower than that in the newly diagnosed group and the ineffective treatment group, but still higher than that in the healthy control group. The reason may be that there are still a small number of residual tumor cells in the body after complete remission, which also indirectly suggests that SF is positively correlated with tumor load. Yamazaki E, et al. [9] found that the SF level of newly diagnosed nasal NK/T-cell lymphoma patients was significantly increased, and the remission rate and overall survival time of patients with elevated SF were significantly lower than those in the non-elevated group. This is consistent with the results of this study. Sivgin S, et al. [10] also studied 165 lymphoma patients receiving hematopoietic stem cell transplantation. It was found that the median survival time of the normal serum ferritin group was significantly higher than that of the elevated serum ferritin group ( 42 months vs 20 months, $\mathrm{P}<0.001$ ), suggesting that the elevated serum ferritin is one of the factors of poor prognosis in these patients.

Iron overload will not only inhibit the formation of erythrocyte colony units and promote erythrocyte apoptosis, but also affect hematopoietic stem cells, destroy hematopoietic microenvironment and affect bone marrow hematopoietic function. After iron overload, it can cause chromosome telomere shortening by inducing oxidative stress, leading to gene instability and the formation of bad chromosomes to accelerate the progress of disease [11]. For patients with significantly elevated SF, iron chelator can be used to remove excess iron and reduce its damage to the body.

In conclusion, $\mathrm{SF}$ level has a certain value in the auxiliary diagnosis of gastric cancer, and it also has a certain guiding significance for the judgment of curative effect and prognosis after treatment. 


\section{Funding}

This work is funded by Natural Science Foundation Project of Anhui Province (Grant number. 1808085MH246).

\section{Conflicts of Interest}

The authors declare no conflicts of interest regarding the publication of this paper.

\section{References}

[1] Bray, F., Ferlay, J., Soerjomataram, I., Siegel, R.L., Torre, L.A. and Jemal, A. (2018) Global Cancer Statistics 2018: GLOBOCAN Estimates of Incidence and Mortality Worldwide for 36 Cancers in 185 Countries. CA: A Cancer Journal for Clinicians, 68, 394-424. https://doi.org/10.3322/caac.21492

[2] The International Non-Hodgkin's Lymphoma Prognostic Factors Project (1993) A Predictive Model for Aggressive Non-Hodgkin's Lymphoma. The New England Journal of Medicine, 329, 987-994. https://doi.org/10.1056/NEJM199309303291402

[3] Zhang, X.Z., Su, A.L., Hu, M.Q., et al. (2014) Elevated Serum Ferritin Levels in Patients with Hematologic Malignancies. Asian Pacific Journal of Cancer Prevention, 15, 6099-6101. https://doi.org/10.7314/APJCP.2014.15.15.6099

[4] Yoh, K.A., Lee, H.S., Park, L.C., et al. (2014) The Prognostic Significance of Elevated Levels of Serum Ferritin before Chemotherapy in Patients with Non-Hodgkin Lymphoma. Clinical Lymphoma, Myeloma and Leukemia, 14, 43-49. https://doi.org/10.1016/j.clml.2013.09.008

[5] Johnston, F.M. and Beckman, M. (2019) Updates on Management of Gastric Cancer. Current Oncology Reports, 21, Article No. 67. https://doi.org/10.1007/s11912-019-0820-4

[6] Alkhateeb, A.A. and Connor, J.R. (2013) The Significance of Ferritin in Cancer: Anti-Oxidation, Inflammation and Tumorigenesis. Biochimica et Biophysica Acta (BBA)-Reviews on Cancer, 1836, 245-254. https://doi.org/10.1016/j.bbcan.2013.07.002

[7] Chen, C.C., Yang, C.F., Yang, M.H., et al. (2005) Pretreatment Prognostic Factors and Treatment Outcome in Elderly Patients with de Novo Acute Myeloid Leukemia. Annals of Oncology, 16, 1366-1373. https://doi.org/10.1093/annonc/mdi259

[8] Yamazaki, E., Tomita, N., Koyama, S., et al. (2014) Serum Ferritin Level Is Prognostic of Patient Outcome in Extranodal NK/T Cell Lymphoma, Nasal Type. Medical Oncology, 31, Article No. 149. https://doi.org/10.1007/s12032-014-0149-7

[9] Sivgin, S., Karamustafaoglu, M.F., Yildizhan, E., et al. (2016) The Prognostic Significance of Elevated Serum Ferritin Levels Prior to Transplantation in Patients with Lymphoma Who Underwent Autologous Hematopoietic Stem Cell Transplantation (autoHSCT): Role of Iron Overload. Clinical Lymphoma, Myeloma and Leukemia, 16, S152-S158. https://doi.org/10.1016/j.clml.2016.02.006

[10] Kepinska, M., Szyller, J. and Milnerowicz, H. (2015) The Influence of Oxidative Stress Induced by Iron on Telomere Length. Environmental Toxicology and Pharmacology, 40, 931-935. https://doi.org/10.1016/j.etap.2015.10.002

[11] Ulbrich, E.J., Lebrecht, A., Schneider, I., et al. (2003) Serum Parameters of Iron Metabolism in Patients with Breast Cancer. Anticancer Research, 23, 5107-5109. 